\title{
2q37.3 Deletion Syndrome: Two Cases with Highly Distinctive Facial Phenotype, Discordant Association with Schizophrenic Psychosis, and Shared Deletion Breakpoint Region on 2q37.3
}

\author{
Yasmin Mehraein $^{\mathrm{a}}$ Martina Pfob $^{\mathrm{a}}$ Ortrud Steinlein $^{\mathrm{a}}$ Eric Aichinger ${ }^{\mathrm{a}}$ \\ Marlene Eggert $^{\mathrm{a}}$ Valerie Bubendorff ${ }^{\mathrm{b}}$ Adelina Mannhart ${ }^{\mathrm{b}}$ Stefan Müller ${ }^{\mathrm{a}}$ \\ anstitute of Human Genetics, University Hospital, Ludwig Maximilian University of Munich, and \\ ${ }^{b}$ kbo - Heckscher-Kinikum gGmbH of Child and Adolescent Psychiatry , Psychosomatics, Psychotherapy, \\ Academic Teaching Hospital of the Ludwig Maximilian University of Munich, Munich, Germany
}

\section{Key Words}

2q37.3 deletion syndrome - Albright hereditary

osteodystrophy - Brachydactyly type E · Schizophrenic psychosis

\begin{abstract}
$2 q 37.3$ deletion syndrome belongs to the chromosomal 2q37 deletion spectrum which clinically resembles Albright hereditary osteodystrophy (AHO) syndrome. It is is mainly characterized by short stature, obesity, round face, brachydactyly type $E$, intellectual disability, behavioral problems, and variable intellectual deficits. Different from classical AHO syndrome, patients with 2 q37 deletion syndrome lack renal parathyroid hormone resistance (pseudohypoparathyroidism) and soft tissue ossification. So far, deletion mapping or molecular breakpoint analyses of 2q37 have been performed in only few patients. Here, we report on 2 patients with 2 q37.3 deletion syndrome. In both patients the breakpoint of the 5.5-Mb terminal microdeletion could be narrowed down to the same $\sim 200-\mathrm{kb}$ interval on 2 q37.3 by BAC-FISH and/or array-CGH. Flanking low-copy repeats may indicate a classical microdeletion syndrome genesis for the 2q37.3 microdeletion subgroup. Clinical evaluation revealed
\end{abstract}

intellectual deficits and type $\mathrm{E}$ brachydactyly typical for classical AHO syndrome together with distinctive facial dysmorphisms not present in the former. Furthermore, one patient presented with schizophrenic psychosis, an observation that would be in accordance with previous reports about an association between schizophrenia susceptibility and an unknown gene within the chromosomal region 2q37.

두 2015 S. Karger AG, Base

Over 115 patients with $2 \mathrm{q} 37$ deletion syndrome have been reported, being the result of either isolated deletion/ microdeletion or monosomy originating from unbalanced translocations [Leroy et al., 2013; Ogura et al., 2014; Jean-Marcais et al., 2015]. The referred symptoms comprise a wide spectrum including intellectual and behavioral, skeletal, cardiac, and urogenital anomalies as well as Wilms tumor, epilepsy, autism, and eczema. Only a fraction of these patients manifest typical symptoms of intellectual disability and brachydactyly type E [Albright hereditary osteodystrophy (AHO)-like phenotype], which is seen in about half of $2 \mathrm{q} 37$ monosomic patients [Leroy et al., 2013]. Since the monogenic brachydactyly mental retardation syndrome (BDMR) is linked

\section{KARGER 125}

(c) 2015 S. Karger AG, Base

$1424-8581 / 15 / 1461-0033 \$ 39.50 / 0$

E-Mail karger@karger.com

www.karger.com/cgr
Yasmin Mehraein

Institute of Human Genetics

University Hospital, Ludwig Maximilan University of Munich

DE-80336 Munich (Germany)

E-Mail Yasmin.Mehraein@ med.uni-muenchen.de 

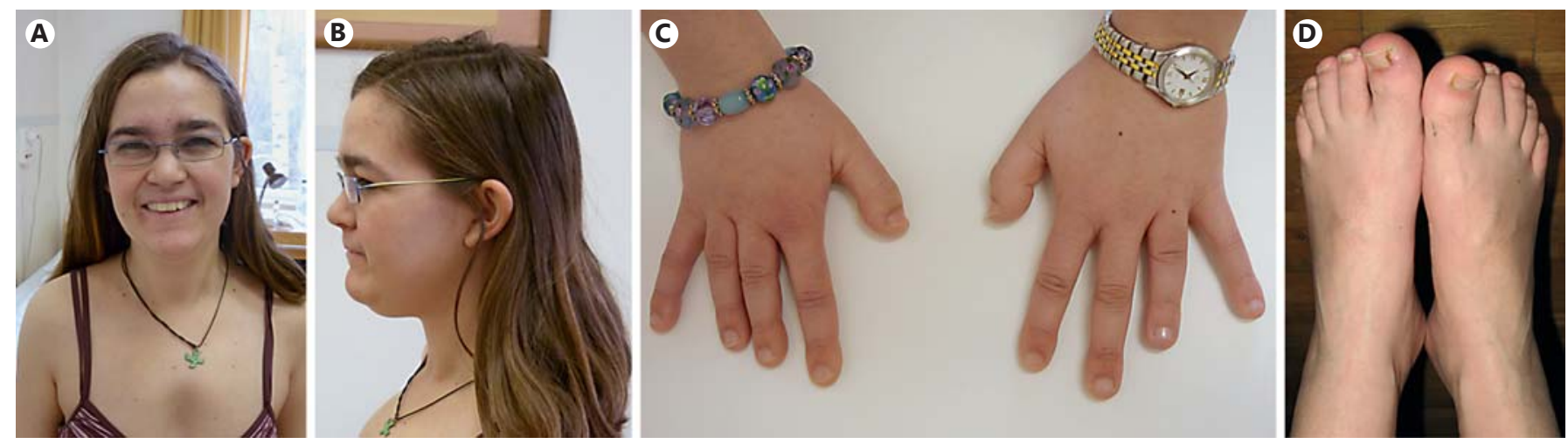

Fig. 1. Case 1. A, B Round face, hypoplastic midface with flat nasal bridge, broad nasal tip, small, deep set eyes, and mildly dysmorphic, deep set ears, facial hypertrichosis, and medial flaring of the bushy eyebrows. C Brachydactyly type $\mathrm{E}$ in both hands with inhomogeneous shortening of phalanges and metacarpals, especially short and broad distal phalanges of the thumbs. D Short toes with no obvious type E brachydactyly in the feet. Side findings: slight partial syndactyly of toes II and III and fingers (C) and toes (D) with very short, everted or ingrown nails. to dominant mutations in the HDAC4 gene on $2 \mathrm{q} 37$, microdeletion syndrome $2 \mathrm{q} 37$ is considered to be a contiguous gene syndrome encompassing the HDAC4 gene as well as possibly several other neighboring genes [Williams et al., 2010].

Although clinical findings are mostly well-documented, molecular analyses of monosomy $2 \mathrm{q} 37$ breakpoints with either FISH or microsatellite panels refining the monosomic region are only performed in a minority of cases. With respect to the variety of symptoms and presumed inter-individual differences in the cytogenetic condition, clear phenotype-genotype conclusions are very limited at present.

Within the $2 \mathrm{q} 37$ deletion spectrum, deletion size as well as deletion origin of the reported cases vary widely, including unbalanced translocations, terminal and interstitial deletions, thus encompassing a rather inhomogeneous molecular cytogenetic group. The 2q37.3 deletion syndrome presents a subclass of the $2 \mathrm{q} 37$ deletion spectrum.

Here, we report on 2 further unrelated cases of $2 \mathrm{q} 37.3$ deletion with the AHO-like phenotype and intriguing facial dysmorphism. Both patients show true interstitial microdeletions assigned to an identical breakpoint in $2 q 37.3$. We could localize the deletion breakpoints within flanking low-copy repeats, being suggestive for a classical microdeletion syndrome genesis [Stankiewicz and Lupski, 2010]. Associated clinical features are described and discussed referring to previous reports of $2 \mathrm{q} 37$ deletion syndrome patients.

\section{Case Report}

Case 1

The 18-year-old woman presented with symptoms of a paranoid schizophrenic psychosis at the psychiatric hospital. Psychotic symptoms had first occurred only few months prior to hospitalization. A mild intellectual impairment with developmental delay and learning disability had been observed since early childhood. Her intelligence had been measured in the lower normal range of learning disability; however, she earned a secondary school degree followed by a 3-year apprenticeship and finished with a diploma.

Facial features were a round face, hypoplastic midface with flat nasal bridge, small, deep set eyes, and mildly dysmorphic, deep set ears. Additionally, facial hypertrichosis and medial flaring of the bushy eyebrows were remarkable (fig. 1A, B). Physical examination showed brachydactyly type $\mathrm{E}$ in both hands with inhomogeneous shortening of phalanges and metacarpals and especially short and broad distal phalanges of the thumbs (fig. 1C). The toes were short, but there was no obvious type E brachydactyly in the feet (fig. 1D). Fingers and toes showed nail anomalies with very short, everted rims or ingrown nails (fig. 1C, D). Further physical features were myopia, pectus excavates, and uterus bicornis.

The family history was unremarkable besides a unilateral broadening and duplication of the terminal phalanx and nail of a thumb in 1 of 2 otherwise inconspicuous elder brothers.

\section{Case 2}

The 32-year-old male patient presented with mild intellectual disability and severe obesity, the latter starting at the age of 12 years. Psychomotor and intellectual delay had required ergotherapy and special schooling. Puberty had been delayed due to hypogonadism leading to testosterone substitution from the age of 15 until the age of 20 . The patient required special school education. Neither was he able to graduate with a degree nor to learn a profession; he was still living at his mother's home and was unable to take responsibility for his own life. 

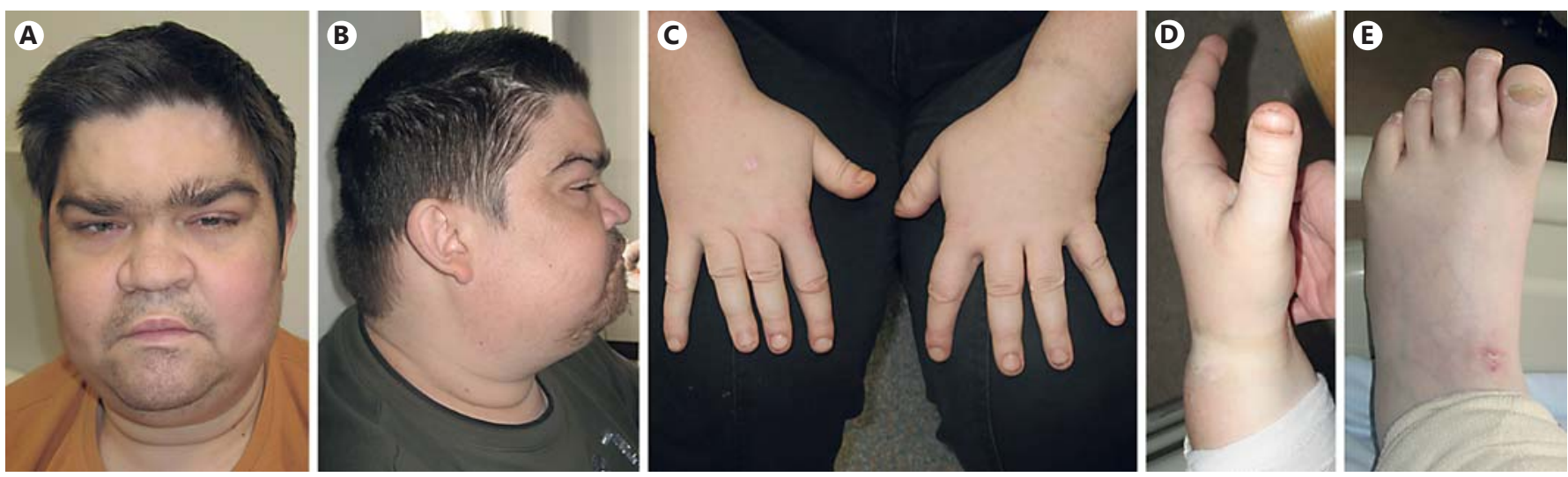

Fig. 2. Case 2. A, B Round face, pronounced midface hypoplasia with flattened nasal bridge, broad nasal tip, deep set eyes with narrow palpebral fissures, hypertelorism, mildly dysmorphic ears, deep frontal hair line, hypertrichosis of the upper facial area, medial flaring of the bushy eyebrows in contrast to the sparse beard growth. C, D Brachydactyly type E with shortening of metacarpal bones III-V as well as various phalanges in both hands. E Feet without brachydactyly but rather small with bilaterally slight shortening of the first metatarsal bone pronounced by the relative overlength of the second toes.
The patient showed striking facial dysmorphism exhibiting a round face, pronounced midface hypoplasia with flattened nasal bridge, deep set small eyes, which appeared almost squinted, hypertelorism, and mildly dysmorphic ears. A deep frontal hair line and general hypertrichosis of the upper facial area were observed; the eyebrows showed medial flaring and appeared rather bushy being in contrast to the sparse beard growth (fig. 2A, B).

Physical examination revealed brachydactyly type $\mathrm{E}$ with shortening of metacarpal bones III-V as well as various phalanges in both hands (fig. 2C, D). The feet did not show apparent brachydactyly but were rather small (5-10th percentile) in comparison to the body length of $180 \mathrm{~cm}$; bilaterally there was a relative overlength of the second toes (fig. 2E). Further skeletal findings comprised additional bone dysplasia with abnormal bending of various long bones especially of the upper limbs (ulnar and radial bending) causing limited mobility and flexion contracture of the elbows. Other physical features were severe obesity with associated skin ulcers (lipedema), micropenis, unilateral testical maldescensus, and sparse pubic hair.

On the primary assumption of AHO syndrome, biochemical Gs-alpha analysis had been performed giving normal results. Family history covering 3 generations was inconspicuous. However, only limited information could be gained. Both parents had reached rather low educational levels, thus mild intellectual deficit or learning disability have not been excluded. One elder brother and one younger half-brother of the maternal side were referred to as unsuspicious and had reached lower school degrees and professional education. Unfortunately, chromosome analyses of the parents and other family members could not be performed.

Within short time after genetic presentation, the patient sustained paraplegia due to vertebral damage caused by an unclear bone marrow inflammation. He died unexpectedly at the age of 34 years as a result of a non-resolved infection.

2q37.3 Deletion Syndrome in Two Cases

\section{Results}

Conventional chromosome analysis on a 550 band level showed a normal 46,XX and 46,XY karyotype in case 1 and 2, respectively. In patient 2, a microdeletion 22q11.2 was excluded by FISH analysis, but FISH using 2q subtelomeric probes $(2 \mathrm{q}$ TelVysion; Abott Molecular) detected a heterozygous terminal microdeletion $2 \mathrm{q} 37$ in both cases. Further iterative BAC-FISH analyses with clones obtained from CHORI (http://bacpac.chori. org) localized the chromosomal breakpoint in $2 \mathrm{q} 37.3$ within an $\sim 200$-kb interval marked by BAC RP11124E11 (chr2:237,550,004-237,731,201, present in both cases) and BAC RP11-713K19 (chr2: 237,699,779237,864,624, deleted in both cases) (hg19, Feb. 2009) (fig. 3). Additional array-CGH analysis (CytoChip Oligo ISCA $4 \times 180 \mathrm{~K}$; BlueGnome, Cambridge, UK) in case 1 revealed a karyotype arr 2q37.3(237,720,284$242,951,149) \times 1$ (Ensembl Release 64), thus confirming the breakpoint region at approximately $237.7 \mathrm{Mb} \pm 100$ $\mathrm{kb}$ (fig. 3).

Conventional chromosome and BAC-FISH analyses in both parents of patient 1 as well as in her brother carrying the thumb duplication revealed a normal chromosomal constitution and excluded the $2 \mathrm{q} 37.3$ microdeletion in each case.

Notably, both the breakpoint-flanking or -spanning BAC clones RP11-124E11 and RP11-713K19 as well as a third, more proximally located BAC, RP11-260J21 (chr2: 237,375,335-237,556,067; hg19), showed cross-hybrid- 


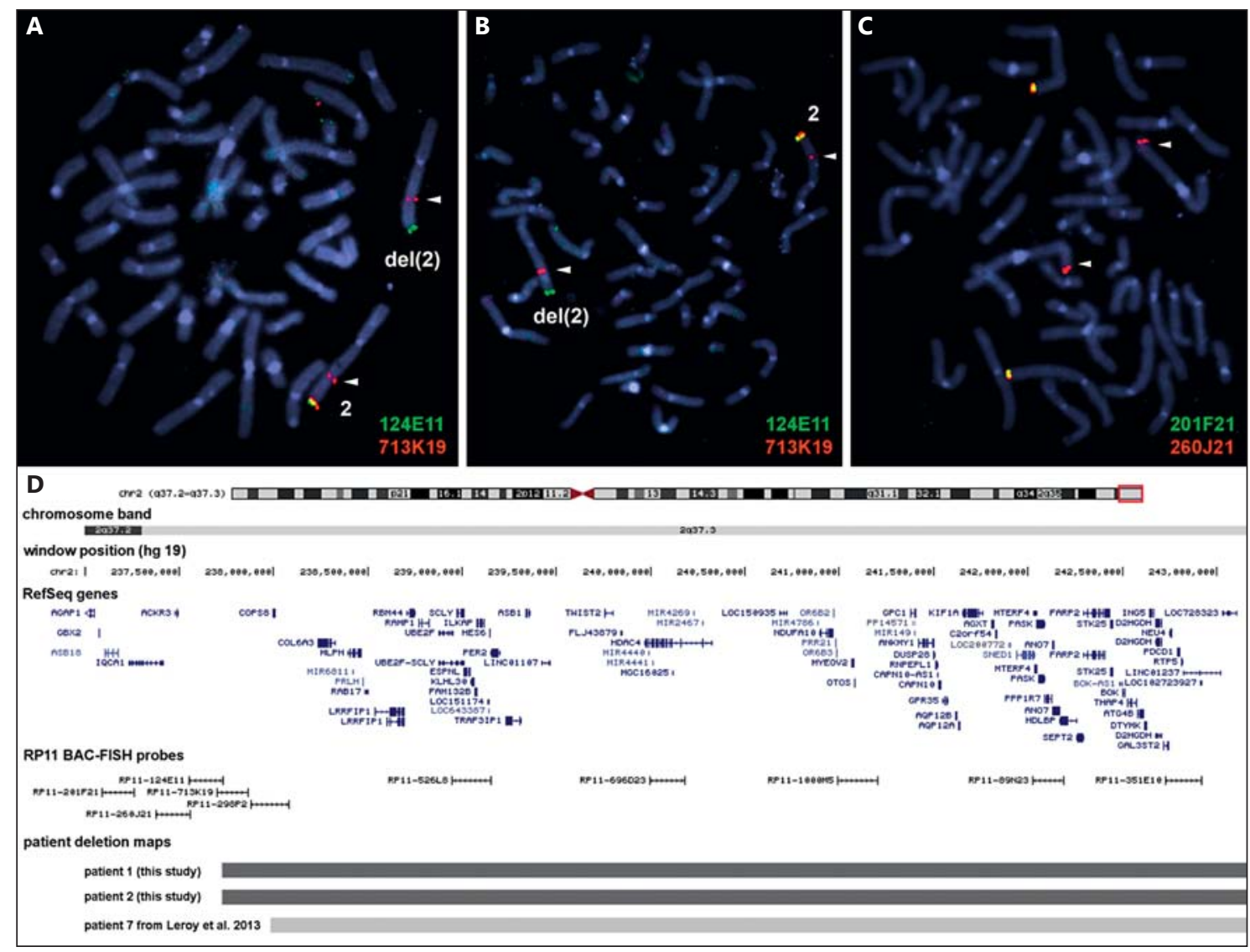

Fig. 3. For case 1 (A) and case 2 (B), FISH experiments localized the deletion breakpoint to an $\sim 200-\mathrm{kb}$ interval on 2q37.3 marked by BAC RP11-124E11 (green, present in both cases) and BAC RP11-713K19 (red, deleted in both cases). In both cases, secondary FISH signals from BAC RP11-713K19 in 2q31 (arrowheads) were visible. C FISH in case 1 using more proximally breakpoint-flanking BACs RP11-201F21 (green) and RP11-260J21 (red), where RP11-260J21 showed cross-hybridization to 1p36, as well as in case 2 (not shown). Cross hybridization of RP11-713K19 and RP11-260J21 suggests the presence of segmental duplications in

ization to regions elsewhere in the genome of the 2 patients, their analyzed relatives, and another unrelated, chromosomally normal individual. These secondary FISH signals may indicate the presence of segmental duplications with at least 1 copy in the breakpoint region. Further support for this interpretation is provided by reports on CNVs in this region (copy number gains nssv581030, 706961, and 707006 of uncertain significance in the Database of Genomic Variants DGV; http:// dgv.tcag.ca). the $2 \mathrm{q} 37.3$ breakpoint region with paralogous blocks elsewhere in the genome. D 2q37 deletion map of patients 1 and 2 from our study together with the position of RefSeq genes in the region and the localization of BAC probes used in our FISH experiments (human Feb. 2009 assembly GRCh37/hg19; http://genome.ucsc.edu). We further included $\operatorname{del}(2)(\mathrm{q} 37.3)$ patient 7 from a previously published study [Leroy et al., 2013] showing a similar deletion breakpoint and the same set of deleted genes as the 2 patients presented here.

\section{Discussion}

Both patients showed a characteristic and intriguingly similar facial phenotype, clinically being the major diagnostic feature in combination with the type E brachydactyly. However, despite of the identical breakpoint region, clinical variability was evident (table 1 ).

The presented cases provide further support for the molecular assignment of clinical symptoms observed in 2q37.3 deletion syndrome.

Based on our patients as well as reported individuals with similar chromosomal breakpoints [Leroy et al., 
Table 1. Comparison of clinical features of our patients with the only patient (P7) described in the literature so far showing a similar breakpoint region

\begin{tabular}{|c|c|c|c|}
\hline & Case 1 & Case 2 & $\begin{array}{l}\text { P7 [Leroy et } \\
\text { al., 2013] }\end{array}$ \\
\hline Psychotic symptoms & + & & \\
\hline Developmental delay & + & + & + \\
\hline Learning disability & + & & \\
\hline Intellectual disability & & + & \\
\hline Hypogonadism & & + & \\
\hline Obesity & & + & + \\
\hline Brachydactyly type E & + & + & \\
\hline Broad distal phalanges of the & & & \\
\hline thumbs & + & + & \\
\hline Small feet & & + & \\
\hline Overlength of the second toes & & + & \\
\hline Short toes & + & + & \\
\hline Bone dysplasia & & + & \\
\hline Nail anomalies & + & & \\
\hline Round face & + & + & \\
\hline Hypoplastic midface & + & + & \\
\hline Flat nasal bridge & + & + & + \\
\hline Small, deep set eyes & + & + & \\
\hline Hypertelorism & & + & \\
\hline Dysmorphic ears & & + & + \\
\hline Facial hypertrichosis & + & + & \\
\hline Deep frontal hairline & & + & + \\
\hline Medial flaring of bushy eyebrows & + & + & + \\
\hline Myopia & + & & \\
\hline Pectus excavatus & + & & \\
\hline Uterus bicornis & + & & \\
\hline Skin ulcers (lipedema) & & + & \\
\hline Micropenis & & + & \\
\hline Unilateral testical maldescensus & & + & \\
\hline Sparse pubic hair & & + & \\
\hline
\end{tabular}

2013], we would like to highlight the characteristic and rather specific facial dysmorphism of $2 \mathrm{q} 37.3$ deletion syndrome. Especially typical features hereby are the round flat face with deep set, small (squinted) eyes, flat nasal root, facial hirsutism, and heavy eyebrows.

Chromosomal deletion or microdeletion of $2 \mathrm{q} 37$ was first reported in AHO-like syndrome by Wilson et al. [1995]. Since then, a number of cases with terminal 2q37 deletions have been reported, varying widely in deletion size and only partly sharing the AHO-like syndrome phenotype. Brachydactyly type $\mathrm{E}$ is one of the main features of AHO-like and BDMR syndrome. The identification of individuals with characteristic findings and point mutations in the HDAC4 gene located on $2 \mathrm{q} 37$ at $240.1 \mathrm{Mb}$ (hg19) could demonstrate that the HDAC4 gene is causative for brachydactyly type E and BDMR [Williams et al.,
2010]. Among reported cases of $2 q 37$ deletion, depending on the deletion size and localization with respect to HDAC4, brachydactyly may be present or absent.

In a series of 20 cases with $2 \mathrm{q} 37$ monosomy of different chromosomal causes, including interstitial and terminal deletions as well as unbalanced chromosome translocations, no common breakpoint had been identified, thus leading to the hypothesis of a genesis other than low-copy repeat mediated [Aldred et al., 2004]. In contrast to this observation, we identified approximately identical breakpoints in 2 AHO-like-syndrome patients. The same breakpoint furthermore had already been reported in at least 1 further AHO-like patient [Leroy et al., 2013], hinting to a distinct chromosomal region in $2 \mathrm{q} 37$ that is structurally prone to chromosomal breakage or deletion. Our observation of secondary FISH signals from the breakpoint-flanking or -spanning probes (fig. $3 \mathrm{~A}-\mathrm{C}$ ) suggests that the deletion breakpoints in the 2 patients presented here could be localized in a region enriched with segmental duplications. It can be speculated that this feature may be associated with increased genomic instability [Stankiewicz and Lupski, 2010], thus representing a possible cause for an increased risk of recurrent chromosome breakage in the subgroup of patients with $2 \mathrm{q} 37$ deletion syndrome with breakpoints at approximately 239-240 Mb (hg 19). Since typical features of the $2 \mathrm{q} 37$ deletion syndrome like facial characteristics, brachydactyly, and variable cognitive impairment were present in all 3 cases of the small $2 \mathrm{q} 37.3$ microdeletion, the $2 \mathrm{q} 37$ core phenotype might be caused by the small interstitial deletion interval. These results hint to the existence of a true, clinically well-defined interstitial microdeletion syndrome $2 \mathrm{q} 37.3$ which is merged up within the larger group of $2 \mathrm{q} 37$ deletion syndrome. This phenomenon is comparable to other microdeletion syndromes like e.g. Xp22.3 microdeletion syndrome, being a specific interstitial microdeletion with distinct phenotypic features encompassed by the large group of different Xp deletion phenotypes [Li et al., 1992; Stankiewicz and Lupski, 2010].

However, despite the shared 200-kb breakpoint interval and common basic phenotype, clinical variability was evident in our patients. While patient 1 did not suffer from signs of generalized osteodystrophy, patient 2 clearly exhibited pronounced osteodystrophic features with extended skeletal dysplasia besides brachydactyly. Obesity was also only seen in patient 2 , whereas sternal deformity was a feature only in patient 1 . The intellectual impairment, though unequivocal when compared to the family, was only mild to moderate in both cases, enabling a simple school degree and professional education in the 
young woman but special school education without degree and without the ability of self-contained living in case 2. Intellectual deficits, osteodystrophy, sternal anomalies, and obesity are reported findings in patients with $2 \mathrm{q} 37$ deletion syndrome showing rather high variability even in familial cases.

Acute schizophrenic psychosis had occurred in one of the microdeletion patients, which in contrast to autism so far has not been described as a feature of $2 \mathrm{q} 37$ deletion spectrum. According to the WHO International Classification of Diseases (ICD) 10 and the Fifth Edition of the Diagnostic and Statistical Manual of Mental Disorders (DSM-V), autism spectrum disorders and schizophrenic psychosis are considered different disease entities, even though increased comorbidity in part of the autism spectrum diseases - Asperger autism but not typical childhood Kanner autism - suggests common basic pathomechanisms [Simonoff et al., 2008; King and Lord, 2011].
The occurrence of schizophrenic psychosis in patient 1 is of special interest, since the chromosomal region $2 \mathrm{q} 37$ previously had been reported as one of several schizophrenia susceptibility loci [Paunio et al., 2001; Wijsman et al., 2003; Klei et al., 2005]. Though the linkage was obtained in small genetic isolates, this might indicate an unknown gene in 2q37.3 being associated with schizophrenia. Taking into account the overall high frequency of schizophrenic psychosis in the general population, coincidental occurrence due to another possibly genetic disposition is as well possible.

Altogether, the presented cases provide further support for the molecular assignment of clinical symptoms observed in $2 \mathrm{q} 37.3$ deletion syndrome. In addition, the $2 q 37.3$ deletion associated phenotype might be extended to a possible susceptibility for schizophrenia, being in accordance with former linkage studies.

\section{References}

Aldred MA, Sanford RO, Thomas NS, Barrow MA, Wilson LC, et al: Molecular analysis of 20 patients with 2q37.3 monosomy: definition of minimum deletion intervals for key phenotypes. J Med Genet 41:433-439 (2004).

-Jean-Marcais N, Decamp M, Gerard M, Ribault $\mathrm{V}$, Andrieux J, et al: The first familial case of inherited 2q37.3 interstitial deletion with isolated skeletal abnormalities including brachydactyly type E and short stature. Am J Med Genet A 167A:185-189 (2015).

-King BH, Lord C: Is schizophrenia on the autism spectrum? Brain Res 1380:34-41 (2011).

-Klei L, Bacanu SA, Myles-Worsley M, Galke B, Xie W, et al: Linkage analysis of a completely ascertained sample of familial schizophrenics and bipolars from Palau, Micronesia. Hum Genet 117:349-356 (2005).

Leroy C, Landais E, Briault S, David A, Tassy O, et al: The 2q37-deletion syndrome: an update of the clinical spectrum including overweight, brachydactyly and behavioural features in 14 new patients. Eur J Hum Genet 21:602-612 (2013).
Li XM, Yen PH, Shapiro LJ: Characterization of a low copy repetitive element S232 involved in the generation of frequent deletions of the distal short arm of the human X chromosome. Nucleic Acids Res 20:1117-1122 (1992).

- Ogura K, Takeshita K, Arakawa C, Shimojima K, Yamamoto T: Neuropsychological profiles of patients with $2 \mathrm{q} 37.3$ deletion associated with developmental dyspraxia. Am J Med Genet B Neuropsychiatr Genet 165B:684-690 (2014).

Paunio T, Ekelund J, Varilo T, Parker A, Hovatta $\mathrm{I}$, et al: Genome-wide scan in a nationwide study sample of schizophrenia families in Finland reveals susceptibility loci on chromosomes $2 \mathrm{q}$ and $5 \mathrm{q}$. Hum Mol Genet 10:30373048 (2001).

Simonoff E, Pickles A, Charman T, Chandler S, Loucas T, Baird G: Psychiatric disorders in children with autism spectrum disorders: prevalence, comorbidity, and associated factors in a population-derived sample. J Am Acad Child Adolesc Psychiatry 47:921-929 (2008).
Stankiewicz P, Lupski JR: Structural variation in the human genome and its role in disease. Annu Rev Med 61:437-455 (2010).

-Wijsman EM, Rosenthal EA, Hall D, Blundell ML, Sobin C, et al: Genome-wide scan in a large complex pedigree with predominantly male schizophrenics from the island of Kosrae: evidence for linkage to chromosome 2q. Mol Psychiatry 8:695-705, 643 (2003).

-Williams SR, Aldred MA, Der Kaloustian VM, Halal F, Gowans G, et al: Haploinsufficiency of HDAC4 causes brachydactyly mental retardation syndrome, with brachydactyly type E, developmental delays, and behavioral problems. Am J Hum Genet 87:219-228 (2010).

Wilson LC, Leverton K, Oude Luttikhuis ME, Oley CA, Flint J, et al: Brachydactyly and mental retardation: an Albright hereditary osteodystrophy-like syndrome localized to 2q37. Am J Hum Genet 56:400-407 (1995). 